\title{
On the Distributions Corresponding to Bounded Operators in the Weyl Quantization
}

\author{
Ingrid Daubechies* \\ Theoretische Natuurkunde, Vrije Universiteit Brussel, Pleinlaan 2, B-1050 Brussel, Belgium
}

\begin{abstract}
Using properties of an integral transform giving directly the matrix elements of a quantum mechanical operator from the corresponding classical function, we restrict the class of distributions corresponding to bounded operators. As a consequence, we can exhibit a class of functions yielding traceclass operators, and give a bound on their trace-norm.
\end{abstract}

\section{Introduction}

In the Weyl quantization procedure classical functions on phase space are "decomposed" by harmonic analysis in exponential functions, which are then replaced by the corresponding "elementary building blocks for operators", i.e. by the Weyl operators. One obtains in this way a map

$$
f \mapsto \mathbf{Q} f
$$

from functions on phase space to operators on a Hilbert space $\mathscr{H}$ carrying an irreducible representation of the Weyl commutation relations. It has been shown in [1] that this map can also be defined using only $f$ (skipping the harmonic analysis step) and replacing the Weyl operators by so-called Wigner operators, which are simply the Weyl operators multiplied by parity.

In both cases (the original Weyl procedure, and the prescription in [1]), the quantization formula written down is to be understood in a formal sense as long as the class of functions considered is not specified. It has been shown $[2,3]$ that the Weyl quantization map (1) is a unitary map from $L^{2}(E)$, the square integrable functions on phase space, to $\mathscr{L}_{\mathrm{HS}}(\mathscr{H})$, the space of Hilbert-Schmidt operators on $\mathscr{H}$ :

$$
\int d v f^{*}(v) g(v)=\operatorname{Tr}\left(\mathbf{Q} f^{*} \mathbf{Q} g\right) .
$$

For $f$ in $L^{1}(E)$, the absolutely integrable functions on phase space, the operator $\mathbf{Q} f$ is compact [4], and for $f$ in the Schwarz space $\mathscr{S}(E)$, the operator $\mathbf{Q} f$ is trace-class

* Wetenschappelijk medewerker bij het Interuniversitair Instituut voor Kernwetenschappen (in het kader van het navorsingsprojekt $21 \mathrm{EN}$ ) 
$[5,6]$; other exact results concerning $\mathbf{Q} f$ which can be considered as pseudodifferential operators can be found in $[6,7]$.

One can now try to extend (2) and associate a distribution $T_{A}$ to any bounded operator $\mathbf{A}$ by putting $[5,8]$

$$
\forall f \in \mathscr{S}(E): T_{A}(f)=\operatorname{Tr}(\mathbf{A Q} f) .
$$

It is clear that this is then an extension of the inverse of the quantization map restricted to the Hilbert-Schmidt operators. We shall prove that these distributions $T_{A}$ lie in the dual of the space $\mathscr{S}^{4 v+1}$, where $\mathscr{S}^{k}$ is defined by

$$
\mathscr{S}^{k}=\left\{f ; f \text { is } C^{k} \text { in } x, p \text { and } \max _{|m| \leqq k} \sup \left|\left(1+|x|^{2}+|p|^{2}\right)^{\frac{k-|m|}{2}} \partial_{x, p}^{[m]} f(x, p)\right|<\infty\right\} .
$$

Moreover one has the following bound:

$$
\forall f \in \mathscr{S}^{4 v+1}:\left|T_{A}(f)\right| \leqq K_{v}\|\mathbf{A}\|\|f\|_{4 v+1}^{S}
$$

with

$$
\begin{aligned}
\|f\|_{k}^{S} & =\max _{|m| \leqq k} \sup \left|\left(1+\frac{|x|^{2}+|p|^{2}}{2}\right)^{\frac{k-|m|}{2}} \partial_{x, p}^{[m]} f(x, p)\right|<\infty \\
K_{v} & =\frac{3 e}{8}(2 \pi)^{v / 2}\left[16 \sqrt{v}(4 v+1) e^{-1}\right]^{4 v+1} \Gamma(2 v) \Gamma^{-1}(4 v),
\end{aligned}
$$

where $v=\frac{1}{2} \operatorname{dim}($ phase space $)=\operatorname{dim} x$-space.

As a consequence, all the functions in $\mathscr{S}^{4 v+1}$ yield trace-class operators, and one can give a bound on their trace-norm:

$$
f \in \mathscr{S}^{4 v+1} \Rightarrow \operatorname{Tr}|\mathbf{Q} f| \leqq K_{v}\|f\|_{4 v+1}^{S} .
$$

In Sect. 2, we give some definitions and notations. We also introduce the integral transform associating directly to any classical function $f$ the matrix elements between coherent states of its quantized $\mathbf{Q} f[9]$. In fact the results we prove are a consequence of the properties of this integral transform which is closely related to the Bargmann integral transform $[10,11]$. These properties will be studied in a deeper and more thorough way elsewhere [12]; we have however tried to make this paper self contained, and to prove all intermediate results leading to our main statement. All this is done in Sect. 3.

\section{Definitions and Notations}

In most of what follows we shall use intrinsic coordinate-free notations. Occasionally however, we shall give explicit $x-p$-notations.

We denote by $E$ the phase space, i.e. an evendimensional real vector space (dimension $2 v, v \in \mathbb{N}_{*}$ ). On this phase space a non degenerate symplectic (i.e. bilinear, antisymmetric) form $\sigma$ is given. Moreover we suppose we have a $\sigma$-allowed complex structure $J$ on $E$, i.e. a complex structure $J: E \rightarrow E(J$ linear, $J^{2}=-\mathbb{1}$ ) preserving the symplectic structure $\sigma$ 
symplectic structure $\sigma$

$$
\forall v, w \in E: \sigma(J v, J w)=\sigma(v, w)
$$

such that

$$
\begin{aligned}
\forall v \in E: \sigma(v, J v) & \geqq 0 \\
\sigma(v, J v) & =0 \Rightarrow v=0 .
\end{aligned}
$$

These last conditions ensure that the bilinear form $s(v, w)=\sigma(v, J w)$ defines a Euclidean structure on $E$.

Occasionally we will denote by $|v|$ the norm on $E$ defined by the Euclidean form $s$ :

$$
|v|=[s(v, v)]^{1 / 2} .
$$

In $x$-p-notation one usually takes

$$
\begin{aligned}
& E=\mathbb{R}^{2 v}=x \text {-space } \oplus p \text {-space } \\
& E \ni v=(x, p) \\
& \sigma\left((x, p),\left(x^{\prime}, p^{\prime}\right)\right)=\frac{1}{2}\left(p x^{\prime}-p^{\prime} x\right) \\
& J((x, p))=(p,-x) \\
& s\left((x, p),\left(x^{\prime}, p^{\prime}\right)\right)=\frac{1}{2}\left(p p^{\prime}+x x^{\prime}\right) \\
& |(x, p)|=\frac{1}{\sqrt{2}} \sqrt{x^{2}+p^{2}} .
\end{aligned}
$$

For any function $f$ on phase space we define its symplectic Fourier transform $\tilde{f}$ by

$$
\tilde{f}(v)=2^{-v} \int d v^{\prime} e^{i \sigma\left(v, v^{\prime}\right)} f\left(v^{\prime}\right) .
$$

It is easy to check that this defines a continuous map from the Schwarz space $\mathscr{S}(E)$ to itself; moreover one can check that, up to a constant factor, the function $\tilde{\tilde{f}}$ is again $f$. The measure $d v$ on $E$ is normalized by the requirement that $\tilde{\tilde{f}}$ be equal to $f$ :

$$
f(v)=2^{-v} \int d v^{\prime} \tilde{f}\left(v^{\prime}\right) e^{i \sigma\left(v, v^{\prime}\right)} .
$$

$\mathscr{H}$ is a separable Hilbert space carrying an irreducible representation of the Weyl commutation relations, i.e. carrying a strongly continuous irreducible family of unitary operators $W(v)$ satisfying

$$
W(a) W(b)=e^{i \sigma(a . b)} W(a+b) .
$$

In terms of these operators the Weyl quantization formula is

$$
\mathbf{Q} f=2^{-v} \int d v \tilde{f}(v) W\left(-\frac{v}{2}\right)
$$

[the functions $e^{i \sigma\left(\cdot, v^{\prime}\right)}$ in (6) have been replaced by their quantization $\left.W\left(-\frac{v^{\prime}}{2}\right)\right]$.

One has

$W(a) \mathbf{Q} f W(-a)=\mathbf{Q}\left(T_{a} f\right) \quad$ with $\quad\left(T_{a} f\right)(v)=f(v-a)$. 
In [1] it was shown that (7) can also be written in the form

$$
\mathbf{Q} f=2^{v} \int d v f(v) W(2 v) \Pi,
$$

where $\Pi$ is the parity operator, which can be intrinsically defined (up to a factor) on $\mathscr{H}$ by

$$
W(v) \Pi=\Pi W(-v) .
$$

In (8) the symplectic Fourier transform is no longer needed.

One can use either (7) or (8) to define the quantization of the harmonic oscillator hamiltonian $h(v)=s(v, v)$. This can be done quite rigorously, although $h$ is not in $\mathscr{S}(E), L^{1}(E)$ or $L^{2}(E)$; one can use e.g. the concept of pseudodifferential operators $[6,7,13]$ : in the notations of $[6] h$ can be seen to be a GLS-symbol of order $\leqq 2$ which is bounded below and hence gives rise, in the Weyl quantization procedure, to a unique semibounded selfadjoint operator on $\mathscr{H}$ which we call $\mathbf{Q} h$.

This $\mathbf{Q} h$ is in fact everybody's quantum harmonic oscillator hamiltonian and has all its usual spectral properties; we denote its normalized ground state by $\Omega$.

The coherent states are defined as displacements of this ground state:

$\forall a \in E: \Omega^{a}=W(a) \Omega$.

Note that for any $a:\left\|\Omega^{a}\right\|=\|\Omega\|=1$.

These coherent states have several very nice properties $[14,15]$, the most important of which is often called "resolution of the identity":

$\forall \varphi \in \mathscr{H}: \phi_{\varphi}$ defined by $\phi_{\varphi}(a)=\left(\Omega^{a}, \varphi\right)$ is in $L^{2}(E)$ :

$$
\phi_{\varphi} \in L^{2}(E)
$$

and

$$
\forall \varphi, \psi: \int d a \overline{\phi_{\varphi}(a)} \phi_{\psi}(a)=(\varphi, \psi)
$$

or

$$
(\varphi, \psi)=\int d a\left(\varphi, \Omega^{a}\right)\left(\Omega^{a}, \psi\right) .
$$

Using this resolution of the identity (9) one easily sees that any (bounded) operator $\mathbf{A}$ on $\mathscr{H}$ is given by an integral operator on the $\phi_{\varphi}$ with kernel $A(a, b)=\left(\Omega^{a}, \mathbf{A} \Omega^{b}\right)$ :

$$
\begin{aligned}
(\varphi, \mathbf{A} \psi) & =\iint d a d b\left(\varphi, \Omega^{a}\right)\left(\Omega^{a}, \mathbf{A} \Omega^{b}\right)\left(\Omega^{b}, \psi\right) \\
& =\iint d a d b \overline{\phi_{\varphi}(a)} A(a, b) \phi_{\varphi}(b) .
\end{aligned}
$$

One can now try to use the formal expression (8) to obtain a direct correspondence, not between $f$ and $\mathbf{Q} f$, but between $f$ and the matrix elements $Q f(a, b)$ of $\mathbf{Q} f$ between coherent states:

$$
\begin{aligned}
Q f(a, b) & =\left(\Omega^{a}, \mathbf{Q} f \Omega^{b}\right)=2^{v} \int d v f(v)\left(\Omega^{a}, W(2 v) \Pi \Omega^{b}\right) \\
& =\int d v f(v)\{a, b \mid v\}
\end{aligned}
$$


with [9]

$$
\begin{aligned}
\{a, b \mid v\}= & 2^{v}\left(\Omega^{a}, W(2 v) \Pi \Omega^{b}\right) \\
= & 2^{v} \exp \{i[\sigma(a, b)+2 \sigma(b, v)+2 \sigma(v, a)] \\
& \left.-\frac{1}{2} s(2 v-a-b, 2 v-a-b)\right\} .
\end{aligned}
$$

Expression (11) for the integral kernel $\{a, b \mid v\}$ was obtained in [9] by explicit calculations in one particular representation of the Weyl commutation relations, but of course it is valid in any representation.

As announced in the introduction, we will make use of the analogy between the integral kernel $\{a, b \mid v\}$ and the Bargmann integral kernel [9]. For the sake of convenience, we therefore also give here the explicit expression of the Bargmann integral kernel:

$$
\begin{gathered}
A(z ; q)=2^{v / 4} \exp \left[-\frac{1}{2}\left(z^{2}+q^{2}\right)+\sqrt{2} z \cdot q\right] \\
\left(q \in \mathbb{R}^{v}, z \in \mathbb{C}^{v}\right)
\end{gathered}
$$

[the constant has been adjusted to our normalization of the measure: we have $\left.\int d v \exp \left(-|v|^{2}\right)=1\right]$.

(10) is still a formal expression. In [12] it will be studied which sense can be given to these formal matrix elements. In any case, since (8) was already rigorously true for $f \in \mathscr{S}$, and since it is obvious that for $f$ in $\mathscr{S}$ the integral over $v$ and the "sandwiching" with $\Omega^{a}, \Omega^{b}$ may be commuted, (10) holds without problems or interpretation modalities for $f$ in $\mathscr{S}$. This is all we shall need here.

\section{Bounded Operators and the Corresponding Distributions}

Writing out (11) explicitly in $x$-p-notation (5) we get

$$
\begin{aligned}
\{a, b \mid v\}= & 2^{v} \exp \left[\frac{i}{2}\left(p_{a} x_{b}-p_{b} x_{a}+2 p_{b} x_{v}-2 p_{v} x_{b}+2 p_{v} x_{a}-2 p_{a} x_{v}\right)\right. \\
& -\left(x_{v}^{2}+p_{v}^{2}\right)+\left\{x_{v}\left(x_{a}+x_{b}\right)+p_{v}\left(p_{a}+p_{b}\right)\right\} \\
& \left.-\frac{1}{4}\left\{\left(x_{a}+x_{b}\right)^{2}+\left(p_{a}+p_{b}\right)^{2}\right\}\right] \\
= & 2^{v} \exp \left[-\frac{1}{2}\left(\sqrt{2} x_{v}-\frac{x_{a}+x_{b}}{\sqrt{2}}\right)^{2}-i \sqrt{2} x_{v} \cdot \frac{p_{a}-p_{b}}{\sqrt{2}}+\frac{i x_{a}+x_{b}}{2} \cdot \frac{p_{a}-p_{b}}{\sqrt{2}}\right] \\
& \cdot \exp \left[-\frac{1}{2}\left(\sqrt{2} p_{v}-\frac{p_{a}+p_{b}}{\sqrt{2}}\right)^{2}-i \sqrt{2} p_{v} \cdot \frac{x_{b}-x_{a}}{\sqrt{2}}+\frac{i}{2} \frac{p_{a}+p_{b}}{\sqrt{2}} \cdot \frac{x_{b}-x_{a}}{\sqrt{2}}\right] .(1
\end{aligned}
$$

But this is in fact, up to a Gaussian factor, a product of two Bargmann integral kernels. Indeed, one has

$$
\begin{aligned}
\mathscr{A}(x, p ; q) & =A\left(\frac{x+i p}{\sqrt{2}}, q\right) \\
& =2^{v / 4} \exp \left[\frac{1}{4}\left(x^{2}+p^{2}\right)\right] \exp \left[-\frac{1}{2}\left((q-x)^{2}+i p q-\frac{i}{2} x p\right)\right] .
\end{aligned}
$$


Comparing (12) with (13) we see now that

$$
\begin{aligned}
\{a, b \mid v\}= & 2^{v / 2} \exp \left[-\frac{1}{4}\left(x_{a}^{2}+p_{a}^{2}+x_{b}^{2}+p_{b}^{2}\right)\right] \mathscr{A}\left(\frac{x_{a}+x_{b}}{\sqrt{2}}, \frac{p_{a}-p_{b}}{\sqrt{2}} ; \sqrt{2} x_{v}\right) \\
& \cdot \mathscr{A}\left(\frac{p_{a}+p_{b}}{\sqrt{2}}, \frac{x_{b}-x_{a}}{\sqrt{2}} ; \sqrt{2} p_{v}\right) \\
= & 2^{v / 2} \exp \left[-\frac{1}{2}\left(\left|c_{a b}\right|^{2}+\left|d_{a b}\right|^{2}\right)\right] A\left(c_{a b} ; \sqrt{2} x_{v}\right) A\left(d_{a b} ; \sqrt{2} p_{v}\right)
\end{aligned}
$$

with

$$
c_{a b}=\frac{1}{\sqrt{2}}\left(x_{a}+x_{b}, p_{a}-p_{b}\right), \quad d_{a b}=\frac{1}{\sqrt{2}}\left(p_{a}+p_{b}, x_{b}-x_{a}\right) .
$$

Note that

$$
\left|c_{a b}\right|^{2}+\left|d_{a b}\right|^{2}=\frac{1}{2}\left(x_{a}^{2}+p_{a}^{2}+x_{b}^{2}+p_{b}^{2}\right)=|a|^{2}+|b|^{2} .
$$

[Here $|a|,|b|,\left|c_{a b}\right|,\left|d_{a b}\right|$ are defined as in (4) or (5).]

The square of the Gaussian occurring in (14) or (15) is exactly the weight occurring in the corresponding measure $d \mu_{\nu}\left(c_{a b}\right) \cdot d \mu_{v}\left(d_{a b}\right)$ in [10].

As a consequence of (15) we can use Bargmann's estimates [11]; in particular

$$
f \in \mathscr{S} \Rightarrow \forall k \in \mathbb{N}:|Q f(a, b)| \leqq a_{k} 2^{-v / 2+k}\|f\|_{k}^{S}\left(1+\left|c_{a b}\right|^{2}+\left|d_{a b}\right|^{2}\right)^{-k / 2}
$$

or

$$
|Q f(a, b)| \leqq a_{k} 2^{-v / 2+k}\|f\|_{k}^{S}\left(1+|a|^{2}+|b|^{2}\right)^{-k / 2}
$$

with

$$
\left.\|f\|_{k}^{S}=\max _{|m| \leqq k} \sup _{v}\left(1+|v|^{2}\right)^{\frac{k-|m|}{2}} \partial^{[m]} f(v)\right\}
$$

Here $a_{k}$ is the constant defined by Bargmann (see [11], Theorem 1.2, (1.18d) with $n$ replaced by $2 v)$ :

$$
a_{k}=\frac{3}{2} e(4 \pi)^{v / 2}(16 v)^{k / 2} \alpha_{k},
$$

with $\alpha_{k}=1$ for $k \leqq 2, \alpha_{k}=\left(e^{-1} k\right)^{k}$ for $k \leqq 3$; the factor $2^{-v / 2+k}$ in (16a) is a conse-

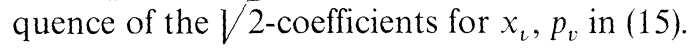

To derive the bounds we aim at, we also need the following two lemmas:

Lemma 1. For any $f$ in $L^{2}(E)$ :

$$
\left(\Omega^{a}, \mathbf{Q} f \Omega^{b}\right)=\int d v f(v)\{a, b \mid v\}
$$

and

$\forall f, g \in L^{2}(E): \iint d a d b \overline{Q f(a, b)} Q g(a, b)=\int d v \overline{f(v)} g(v)$.

Proof. We know already that (17) holds for $f \in \mathscr{S}$. 
On the other hand, if we put $\forall f \in L^{2}(E): \varphi_{f}(a, b)=\int d v\{a, b \mid v\} f(v)$, then we know from (15) and [9] that the map $f \mapsto \varphi_{f}$ is isometric from $L^{2}(E)$ to $L^{2}(E \times E)$ :

$\iint d a d b \overline{\varphi_{f}(a, b)} \varphi_{g}(a, b)=\int d v \overline{f(v)} g(v)$.

Take now $f \in L^{2}(E)$, and $f_{n} \in \mathscr{S}(E)$ such that $f_{n} \stackrel{L^{2}}{\longrightarrow} f$.

One easily can show from the similar statement in [9] that $L^{2}$-convergence of a sequence $f_{n}$ in $L^{2}(E)$ implies pointwise convergence of the $\varphi_{f_{n}}$. So

$$
\varphi_{f}(a, b)=\lim _{n \rightarrow \infty} \varphi_{f_{n}}(a, b)=\lim _{n \rightarrow \infty} Q f_{n}(a, b) .
$$

On the other hand $f_{n} \stackrel{L^{2}}{\longrightarrow} f$ implies $\mathbf{Q} f_{n} \stackrel{\text { Hilb.Schm. }}{\longrightarrow} \mathbf{Q} f$, hence

$$
\begin{aligned}
\forall a, b: Q f(a, b) & =\left(\Omega^{a}, \mathbf{Q} f \Omega^{b}\right)=\lim _{n \rightarrow \infty}\left(\Omega^{a}, \mathbf{Q} f_{n} \Omega^{b}\right) \\
& =\lim _{n \rightarrow \infty} Q f_{n}(a, b)=\varphi_{f}(a, b) .
\end{aligned}
$$

Statements (17) and (18) follow now directly from $Q f(a, b)=\varphi_{f}(a, b)$, the definition of $\varphi_{f}$ and relation (19).

We use these results to prove the following lemma.

Lemma 2. For any $\mathbf{B}$ trace-class on $\mathscr{H}$, the function $\psi_{B}(b)=B(b, b)=\left(\Omega^{b}, \mathbf{B} \Omega^{b}\right)$ is in $L^{1}(E)$ and

$$
\operatorname{Tr} \mathbf{B}=\int d b \psi_{B}(b)=\int d b\left(\Omega^{b}, \mathbf{B} \Omega^{b}\right) .
$$

Proof. Let $\mathbf{B}$ be a trace-class operator on $\mathscr{H}$. There exist $\mathbf{C}, \mathbf{D}$ Hilbert-Schmidt operators such that $\mathbf{B}=\mathbf{C}^{*} \cdot \mathbf{D}$.

Because of the unitarity of the Weyl correspondence between $L^{2}(E)$ and $\mathscr{L}_{\mathrm{HS}}(\mathscr{H})$ [3], there exist $f, g$ such that $\mathbf{C}=\mathbf{Q} f, \mathbf{D}=\mathbf{Q} g$. Hence

$$
\begin{aligned}
\psi_{B}(b) & =\left(\Omega^{b}, \mathbf{Q} f^{*} \mathbf{Q} g \Omega^{b}\right) \\
& =\int d a\left(\Omega^{b}, \mathbf{Q} f^{*} \Omega^{a}\right)\left(\Omega^{a}, \mathbf{Q} g \Omega^{b}\right) .
\end{aligned}
$$

Since $Q f^{*}(b, a), Q g(a, b)$ are both in $L^{2}(E \times E), \psi_{B}$ is clearly in $L^{1}(E)$. Moreover, using (2) we get

$$
\begin{aligned}
& \operatorname{Tr} \mathbf{B}=\operatorname{Tr}\left(\mathbf{Q} f^{*} \mathbf{Q} g\right)=\int d v \overline{f(v)} g(v) \\
& =[\text { by (18) }] \iint d a d b \overline{\left(\Omega^{a}, \mathbf{Q} f \Omega^{b}\right)}\left(\Omega^{a}, \mathbf{Q} g \Omega^{b}\right) \\
& =\iint \operatorname{dadb}\left(\Omega^{b}, \mathbf{Q} f^{*} \Omega^{a}\right)\left(\Omega^{a}, \mathbf{Q} g \Omega^{b}\right) \\
& =\int d b \psi_{B}(b) \text {. }
\end{aligned}
$$

With the help of these two lemmas and the bounds (16), we shall be able to prove our main statement.

Generalizing (2), we define for any operator $\mathbf{A} \in \mathscr{B}(\mathscr{H})$ the corresponding distribution $T_{A}$ as $[5,8]$

$$
T_{A}(f)=\operatorname{Tr}(\mathbf{A Q} f) \text { for any } f \in \mathscr{S} .
$$

This makes sense: for $f \in \mathscr{S}, \mathbf{Q} f$ is trace-class [5,6], and since $\mathbf{A}$ is bounded, $\mathbf{A Q} f$ is trace-class, too.

We have now: 
Theorem. For any bounded operator $\mathbf{A}$ the distribution $T_{A}$ satisfies the following bound:

$$
\forall f \in \mathscr{S}:\left|T_{A}(f)\right| \leqq K_{v}\|\mathbf{A}\| \cdot\|f\|_{4 v+1}^{S},
$$

where $K_{v}$ is a constant depending only on $v$, and $\|f\|_{4 v+1}^{S}$ is as defined in (16b).

Proof. Applying Lemma 2 and the definition of $T_{A}$ we get

$$
T_{A}(f)=\int d b\left(\Omega^{b}, \mathbf{A Q} f \Omega^{b}\right) .
$$

By (16a) for $k$ big enough, $Q f(a, b)$ is certainly in $L^{1}(E \times E)$, which implies we can use the "resolution of the identity" (9) without any qualms in (22):

$$
T_{A}(f)=\iint d a d b\left(\Omega^{b}, \mathbf{A} \Omega^{a}\right)\left(\Omega^{a}, \mathbf{Q} f \Omega^{b}\right) .
$$

Hence

$$
\begin{aligned}
\mid T_{A}(f) & \leqq \sup _{a, b}\left|\left(\Omega^{b}, \mathbf{A} \Omega^{a}\right)\right| \iint \operatorname{dad} b|\mathbf{Q} f(a, b)| \\
& \leqq\|\mathbf{A}\| a_{4 v+1} 2^{7 v / 2+1}\|f\|_{4 v+1}^{S} \iint d a d b\left(1+|a|^{2}+|b|^{2}\right)^{-\frac{4 v+1}{2}} .
\end{aligned}
$$

So $(21)$ is satisfied with

$$
\begin{aligned}
K_{v} & =2^{7 v / 2+1} a_{4 v+1} \int d^{4 v} x \frac{1}{\left(1+x^{2}\right)^{\frac{4 v+1}{2}}} \cdot \pi^{-2 v} \\
& =\frac{3 e}{8}(2 \pi)^{v / 2}\left(16 \sqrt{v}(4 v+1) e^{-1}\right)^{4 v+1} \Gamma(2 v) \Gamma^{-1}(4 v) .
\end{aligned}
$$

Since the topology on $\mathscr{S}$ is completely given by the set of norms \|\|$_{k}^{S}$, (21) gives us indeed a bound on $T_{A}$ in $\mathscr{S}^{\prime}$. Note that this bound can be refined if we have some a priori knowledge of the decay of $A(a, b)$ for $a, b \rightarrow \infty$, or that, on the other hand, bounds can also be given on $T_{A}$ for $\mathbf{A}$ unbounded, but with controlled growth of $A(a, b)$ for $a, b \rightarrow \infty$. Note also that in fact (21) enables us to consider the distributions $T_{A}$ as elements of the dual of $\mathscr{S}^{4 v+1}$, the closure of $\mathscr{S}$ w.r.t. the norm \|\|$_{4 v+1}^{S}$.

As a consequence of the theorem we have

Corollary. Let $\mathscr{S}^{k}$ be the function space

$$
\mathscr{S}^{k}=\left\{f: E \rightarrow \mathbb{C} ; f \text { is } C^{k} \text { and }\|f\|_{k}^{S}=\max _{|m| \leqq k} \sup \left|\left(1+|v|^{2}\right)^{\frac{k-|m|}{2}} \partial^{[m]} f(v)\right|<\infty\right\} .
$$

Then for any $f$ in $\mathscr{S}^{4 v+1}$, the operator $\mathbf{Q} f$ is trace-class and

$$
\|\mathbf{Q} f\|_{1}=\operatorname{Tr}(|\mathbf{Q} f|) \leqq K_{v}\|f\|_{4 v+1}^{S} .
$$

Proof. Take $f$ in $\mathscr{S}$. By the polar decomposition of $\mathbf{Q} f$ one has $\mathbf{Q} f=\mathbf{U}|\mathbf{Q} f|$ for some partial isometry $\mathbf{U}$.

Since $\mathbf{U}^{*} \mathbf{U}_{\operatorname{Ran}|\mathbf{Q} f|}=\left.\mathbb{1}\right|_{\operatorname{Ran}|\mathbf{Q} f|}$, we have also

$$
|\mathbf{Q} f|=\mathbf{U}^{*} \mathbf{Q} f \text {. }
$$


Hence

$$
\begin{aligned}
\|\mathbf{Q} f\|_{1} & =\operatorname{Tr}|\mathbf{Q} f| \leqq K_{v}\left\|\mathbf{U}^{*}\right\|\|f\|_{4 v+1}^{S} \\
& \leqq K_{v}\|f\|_{4 v+1}^{S} .
\end{aligned}
$$

Since $\mathscr{S}$ is dense in $\mathscr{S}^{4 v+1}$ w.r.t. the norm \|\|$_{4 v+1}^{S}$, since the integral transform with kernel $\{a, b \mid v\}$ obviously makes sense on $\mathscr{S}^{4 v+1}$ and gives rise to bounded operator kernels $\left(\mathscr{S}^{4 v+1} C L^{2}(E)\right)$, we can extend (24) to all functions in $\mathscr{S}^{4 v+1}$.

Remark. Since the \|\|$_{k}^{S}$-norms form a totally ordered set and determine completely the topology on $\mathscr{S}$, and since it was proven in [5] that the map $\mathbf{Q}$, when restricted to $\mathscr{S}$, is continuous from $\mathscr{S}$ to the trace-class operators, a bound of the kind (23) was to be expected. To our knowledge, however, this is the first time a precise bound of this kind (with $k$ and the constant $K_{v}$ explicitly given) is derived.

\section{Concluding Remarks}

We have derived a condition (21) for distributions to correspond to bounded operators in the Weyl quantization. It is obvious that the condition is not sufficient : the harmonic oscillator hamiltonian for instance does satisfy a bound of the kind (21), but does definitely not give rise to a bounded operator. On the other hand, though the bound may be rather coarse, it is a better characterization of the image under the inverse Weyl transform of the bounded operators than was known (at least to our knowledge).

We are conscious of the fact that the analogy with the Bargmann integral transform of the integral transform with kernel $\{a, b \mid v\}$ has been far from fully exploited here; as we indicated, whole families of bounds can be derived from it. In fact the way in which we proceded here is only one of the possibilities to extract useful information out of the study of the integral transform, and bounds can be derived for even more general objects than distributions. All this will be developed elsewhere [12]: our aim here was only to derive this one bound on the class of distributions on phase space corresponding to bounded operators on the Hilbert space.

Note. In fact sharper results than those in (21) or (23) can be derived using a family of Hilbert spaces $\mathscr{H}^{\varrho}$ instead of the Banach spaces $\mathscr{S}^{k}$. We give here only the results; the derivation of these results can be found in [12] or [16].

Define $\mathrm{H}_{2}$ to be the operator in $L^{2}(E)$

$$
\begin{aligned}
H_{2} & =2|v|^{2}-\frac{1}{8} \Delta_{v} \\
& =\left(\text { in } x-p \text {-notations) } x^{2}+p^{2}-\frac{1}{4} \Delta_{x}-\frac{1}{4} \Delta_{p} .\right.
\end{aligned}
$$

For any $\varrho$ in $\mathbb{R}$, let $\mathscr{H}^{\varrho}$ be the Hilbert space defined by the completion of $\mathscr{S}(E)$ with respect to the norm

$$
|f|_{\varrho}=\left\|\left(H_{2}+v\right)^{\varrho / 2} f\right\|_{L^{2}} .
$$

One has then the following results:

$$
\forall \varepsilon>0: f \in \mathscr{H}^{2 v+\varepsilon} \Rightarrow Q f \quad \text { trace-class }
$$


and

$$
\begin{aligned}
\operatorname{Tr}|Q f| & \leqq\left(\frac{\zeta(1+\varepsilon)}{\Gamma(2 v)}\right)^{1 / 2}|f|_{2 v+\varepsilon}, \\
A \in \mathscr{B}(\mathscr{H}) & \Rightarrow T_{A} \in \mathscr{H}^{-2 v-\varepsilon}
\end{aligned}
$$

and

$$
\left|T_{A}\right|_{-2 v-\varepsilon} \leqq\left(\frac{\zeta(1+\varepsilon)}{\Gamma(2 v)}\right)^{1 / 2}\|A\|,
$$

where $\zeta$ is Riemann's zeta-function.

Since one can easily check that $\mathscr{S}^{3 v+3} C \mathscr{H}^{2 v+2}$, and $4 v+1>3 v+3$ for $v>2$, these results are indeed sharper than (21), (23).

Acknowledgement. The constant encouragement by and interest in the author's work of Prof. A. Grossmann are gratefully acknowledged.

\section{References}

1. Grossmann, A.: Commun. Math. Phys. 48, 191-194 (1976)

2. Segal, I.E.: Math. Scand. 13, 31 (1963)

3. Pool, J.C.: J. Math. Phys. 7, 66-76 (1966)

4. Loupias, G.: Ph. D. Thesis, Univ. d'Aix, Marseille, 1966

5. Loupias, G., Miracle-Sole, S.: Ann. Inst. H. Poincaré 6, 39-58 (1967)

6. Voros, A.: J. Funct. Anal. 29, 104-132 (1978)

7. Berezin, F.A., Šubin, M.A.: Coll. Math. Soc. J. Bolyai 5, 21-52, Tihany (Hungary), 1970

8. Grossmann, A.: Momentum-like constants of motion. In: Statistical mechanics and field theory (eds. R. N. Sen and C. Weil). Halstead 1972

9. Daubechies, I., Grossmann, A. : An integral transform related to quantization. To be published in J. Math. Phys.

10. Bargmann, V.: Comm. Pure Appl. Math. 14, 187-214 (1961)

11. Bargmann, V.: Comm. Pure Appl. Math. 20, 1-101 (1967)

12. Daubechies, I., Grossmann, A.: In preparation

13. Grossmann, A., Loupias, G., Stein, E.M.: Ann. Inst. Fourier 23, 343-368 (1969)

14. Klauder, J.R.: Ann. Phys. 11, 123-168 (1960)

15. Klauder, J.R.. Sudarshan, E.C.: Fundamentals of quantum optics. New York: Benjamin 1968

16. Daubechies, I.: Ph. D. Thesis. VUB Brussels 1980

Communicated by J. Ginibre

Received March 3, 1980 